\title{
Estudo Estrutural de Complexo de Cobre(II) com Ligante Hidrazona
}

Eduardo de Assis Duarte*1(IC), Pedro H. Santiago²(PG), João Batista L. Martins ${ }^{1}(\mathrm{PQ})$, Claudia Cristina Gatto ${ }^{2}(\mathrm{PQ}) *$ *duduarte3@gmail.com

${ }^{1}$ LQC - Laboratório de Química Computacional - UnB

${ }^{2}$ LASIC - Laboratório de Síntese Inorgânica e Cristalografia - UnB

Palavras-chave: Composto de Coordenação, Estudo Teórico, Raios-X, Gaussian09.

\section{Introdução}

As hidrazonas constituem uma classe de compostos que apresentam complexas propriedades biológicas, dentre elas, antitumoral, antituberculose, antifúngicas e ainda anticonvulsionantes. ${ }^{1}$ Esses compostos são frequentemente utilizados como ligantes, na área de química de coordenação, uma vez que seus complexos metálicos apresentam propriedades biológicas acentuadas. ${ }^{2}$ Assim, a busca por complexos que potencializem essas propriedades é um campo que está em constante expansão. ${ }^{3}$

Os estudos teóricos por meio da química computacional se tornaram um complemento bastante útil aos estudos experimentais, principalmente com $\mathrm{o}$ desenvolvimento de métodos cada vez mais sofisticados, algoritmos mais bem desenvolvidos, compiladores mais específicos e eficientes, além de computadores cada dia mais potentes.

\section{Metodologia}

A princípio, a estrutura molecular do composto de coordenação $\left[\mathrm{Cu}_{2}(\mathrm{apbh})_{2}(\mathrm{DMF})\left(\mu-\mathrm{SO}_{4}\right)\right]$ (Figura 1) foi modelada de acordo com os dados .CIF, obtidos após as análises experimentais de difração de Raios-X de monocristal do complexo.

Os cálculos de otimização foram feitos realizados pelo método de ondas planas do VASP e pelo Gaussian09, através da Teoria do Funcional de Densidade. Foram avaliadas além da geometria, o espectro vibracional no infravermelho e ultravioleta, além dos cálculos das cargas e dos mapas eletrostáticos.

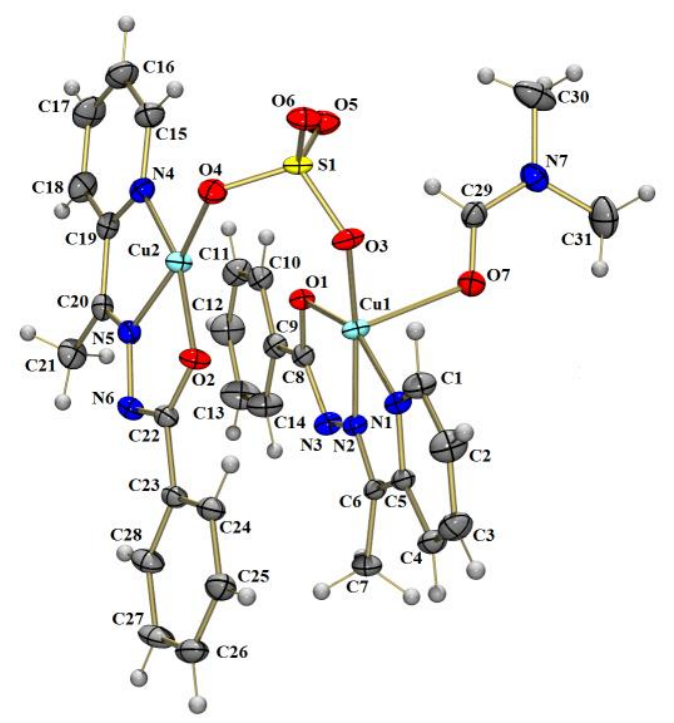

Figura 1. Ilustração da unidade assimétrica do complexo $\left[\mathrm{Cu}_{2}(\mathrm{apbh})_{2}(\mathrm{DMF})\left(\mu-\mathrm{SO}_{4}\right)\right]$. 


\section{Resultados e Discussão}

Nessa etapa foram utilizados os seguintes funcionais de troca e correlação B3LYP, PBE1PBE e B3PW91 (híbridos), CAM-B3LYP (híbrido de longo alcance) e wB97-XD (que inclui dispersão empírica), para todos esses funcionais foram utilizadas as bases atômicas LANL2DZ para o Cobre e 6-31G(d,p) para todos os demais átomos.

Depois de otimizada a estrutura, foram feitos os cálculos de desvio médio quadrático (RMSD), para que fosse feito o comparativo entre os dados teóricos obtidos com os experimentais. Esse resultado foi disposto na Tabela 1, abaixo.

Tabela 1. Comparativo dos RMSD em relação ao experimental (A)

\begin{tabular}{|c|c|c|c|c|c|}
\hline VASP (PW91) & B3PW91 & B3LYP & wB97-XD & PBE1PBE & CAM-B3LYP \\
\hline 0,1412 & 0,3996 & 0,3761 & 0,9482 & 0,2666 & 0,2987 \\
\hline
\end{tabular}

Utilizando a geometria otimizada, foram calculados os espectros vibracional no infravermelho e de absorção no ultravioleta-visível. Os resultados mais interessantes foram resumidos nas Tabelas 2 e 3, mostradas logo abaixo.

Tabela 2. Comparativo das bandas no espectro de infravermelho $\left(\mathrm{cm}^{-1}\right)$

\begin{tabular}{|c|c|c|c|c|c|c|c|}
\hline & $\mathbf{v}(\mathbf{N}-\mathbf{H})$ & $\mathbf{v}(\mathbf{C}=\mathbf{O})$ & $\mathbf{v}(\mathbf{C}=\mathbf{N})$ & $\mathbf{v}\left(\mathbf{C}=\mathbf{N}_{\mathbf{p y}}\right)$ & $\mathbf{v}(\mathbf{C}-\mathbf{O})$ & $\mathbf{v}(\mathbf{N}-\mathbf{N})$ & $\boldsymbol{\delta}(\mathbf{p y})$ \\
\hline Experimental & ---- & 1641 & 1599 & 1566 & 1372 & 1120 & 713 \\
\hline Calculado & ---- & 1637 & 1594 & 1556 & 1395 & 1164 & 729 \\
\hline
\end{tabular}

Tabela 3. Comparativo das absorções no espectro de UV-Vis (nm)

\begin{tabular}{|c|c|c|c|}
\hline & $\boldsymbol{\pi} \rightarrow \boldsymbol{\pi}^{*}$ & TCLM & d-d \\
\hline Experimental & 261 & 381 & 683 \\
\hline Calculado & 255 & 380 & 683 \\
\hline Força do & 0,0647 & 0,2033 & 0,0037 \\
Oscilador & & & \\
\hline
\end{tabular}

\section{Conclusão}

Os resultados obtidos foram satisfatórios, tendo em vista que os métodos computacionais aplicados ao composto de coordenação estudado geraram resultados dentro do que era esperado, tanto para as análises de infravermelho, ultravioleta-visível quanto nos cálculos dos mapas de potencial eletrostático por NBO. Portanto, os resultados alcançados foram positivos, complementando e corroborando os dados previamente obtidos pelas técnicas experimentais de análise.

\section{Referências}

${ }^{1}$ El-Sabbagh, O. I., Rady, H. M., Eur. J. Med. Chem. 2009, 3680, 44.

${ }^{2}$ Sharma, R. N., Sharma K. P., Dixit, S. N., Asian J. Chem. 2010, 7683, 22.

${ }^{3}$ Tiago S. F., Santiago, P. H. O., Amaral, M. M. P., Martins, J. B. L., J. Coord. Chem. 2015, 69, 330

\section{Agradecimentos}

Agradecimentos ao suporte computacional do LQC, aos dados experimentais do LASIC, à FAP-DF, ao CNPq e ao IQ-UnB. 